\title{
GRBs in the Era of Rapid Response Telescopes
}

\author{
Neil Gehrels* \\ Astroparticle Physics Division, NASA/Goddard Space Flight Center, Greenbelt, MD 20771, USA \\ E-mail: neil.gehrels@nasa.gov \\ John K. Cannizzo \\ CRESST/Joint Center for Astrophysics, Univ. of Maryland, Baltimore County, Baltimore, MD \\ 21250, USA \\ E-mail: john.k.cannizzo@nasa.gov
}

\begin{abstract}
NASA's Swift and Fermi satellites continue to chase gamma-ray bursts (GRBs) and make groundbreaking discoveries. The resulting science has been greatly enhanced by follow-up studies and coincident event searches from a variety of observatories, including RAPTOR, Watcher, REM, Pi of the Sky, MASTER, iPTF, GROND, GTC, RATIR, DCT, Asiago, Nanshan, Liverpool Robotic Telescope, KAIT, NOT, Gemini, Magellan, Keck, VLT, VLA, ACTA, ALMA, AMI, WSRT, GMRT, CARMA, LOFAR, HAWC, VERITAS, MAGIC, HESS, IceCube, ANTARES, LIGO, HST, Chandra, Spitzer, Fermi, AGILE, INTEGRAL, NUSTAR, IPN, MAXI, CALET, and AstroSAT. These observatories have added to our knowledge of the early-time behavior of GRBs, such as the short-lived reverse-shock which has been seen now in a small handful of the brightest bursts. The emphasis on longer term optical observations, days to weeks after the burst, has increased with the prospects in a few years for multiple active gravitational wave observatories providing good localizations for compact object mergers, and the potential for seeing the isotropic kilonova emission if one of the objects is a neutron star.
\end{abstract}

4th Annual Conference on High Energy Astrophysics in Southern Africa

25-27 August, 2016

Cape Town, South Africa

\footnotetext{
* Speaker.
} 


\section{Catching GRBs in the Act}

The most powerful transient events in the universe observed to date are merging black hole binaries (Abbott et al. 2016ab) for which the luminosity in gravitational waves in the final $\sim 30 \mathrm{~ms}$ is $\sim 10-100$ times that of all the $\sim 10^{22}$ stars in the universe. The total energy released is $\gtrsim M_{\odot} c^{2}$ $\left(=1.8 \times 10^{54} \mathrm{erg}\right)$. GRBs are the second most powerful transients, with isotropic equivalent energy (i.e., estimated from beamed flux) in some cases approaching $M_{\odot} c^{2}$. The actual energy involved is less by the reciprocal of the beaming factor $\sim 10-300$. GRBs are thought to result from either the catastrophic core-collapse of massive stars, or from compact object mergers involving at least one neutron star, i.e., NS-NS or BH-NS (Piran \& Fan 2007; Gehrels, Ramirez-Ruiz, \& Fox 2009). GRBs are found via their "prompt" $\gamma$-ray emission which lasts $\sim 0.1-100 \mathrm{~s}$. Since this bandpass is blocked by Earth's atmosphere, these discoveries must be made by space-based missions carrying $\gamma$-ray detectors with localization capability. GRB counterparts, "afterglows", can appear later in the X-ray, optical, and radio bandpasses after the prompt emission has faded. X-ray afterglow is nearly always seen; optical and radio afterglows are rarer. Afterglow can encompass a wide range of time scales - minutes, days, and even months post-burst. For a small handful of GRBs, one sees optical emission at the same time as the prompt emission.

Rapid GRB follow-up observations are crucial for detecting early optical afterglow. The fireball model accounts for the afterglow peak as being either due to a forward shock passing through the circumstellar environment of the GRB, or a reverse shock moving backwards in the ejecta frame through the GRB jet plasma itself (Zhang \& Kobayashi 2005; Piran \& Fan 2007). Reverse shock emission lasts only a short time due to the short crossing time of the GRB ejecta. Since the reverse shock propagates backwards from the contact discontinuity, the emission peaks at energies lower by a factor of $\Gamma^{2}$ compared to the forward shock, where $\Gamma$ is the bulk Lorentz factor of the flow. However, the luminosity is greater by a factor $\sim \Gamma$ since the reverse shock propagates into the jet, which has a higher density of the seed electrons responsible for the synchrotron emission. The reverse shock emission can reveal properties of the jet, such as baryon loading. In addition, the ratio of the peak fluxes of the spectral components associated with forward and reverse shocks gives an estimate of the jet magnetization (Harrison \& Kobayashi 2013; Laskar et al. 2013).

These basic physical considerations demonstrate the necessity of having both space-based missions and ground-based rapid response observatories in order to study effectively the early optical afterglow, and thereby to gain potential insight into the physics of the forward and reverse shocks.

\subsection{Space Observatories}

\subsubsection{Swift - A Time Domain Observatory}

Swift (Gehrels et al. 2004) carries three instruments, a wide-field Burst Alert Telescope (BAT; Barthelmy et al. 2005) that detects GRBs and positions them to arcminute accuracy, the narrowfield X-Ray Telescope (XRT; Burrows et al. 2005), and the UV-Optical Telescope (UVOT; Roming et al. 2005). BAT is a coded aperture hard X-ray $(15-350 \mathrm{keV})$ imager with $0.5 \mathrm{~m}^{2}$ of CdZnTe detectors (32,000 individual sensors; $\sim 2400 \mathrm{~cm}^{2}$ effective area at $20 \mathrm{keV}$ including mask occultation) and a 1.4 sr half-coded field of view (FOV). XRT is a Wolter 1 grazing incidence, imaging X-ray telescope with a $0.2-10 \mathrm{keV}$ energy range, $120 \mathrm{~cm}^{2}$ effective area at $1.5 \mathrm{keV}, 23^{\prime} .6 \times 23^{\prime} .6 \mathrm{FOV}$, 
point spread function (PSF) half-power diameter of $18^{\prime \prime}$ ( $7^{\prime \prime}$ FWHM), and sensitivity of approximately $2 \times 10^{-14} \mathrm{erg} \mathrm{cm}^{-2} \mathrm{~s}^{-1}$ in $10^{4} \mathrm{~s}$. The UVOT is a modified Ritchey-Chrétien reflector with $30 \mathrm{~cm}$ aperture, $170-650 \mathrm{~nm}$ wavelength range, $17^{\prime} \times 17^{\prime}$ FOV, PSF FWHM of $1.9^{\prime \prime}$ at $350 \mathrm{~nm}$, and sensitivity of $23^{\text {rd }}$ magnitude in white light in $10^{3} \mathrm{~s}$. The Swift mission was built by an international team from the US, UK, and Italy, with contributions also from Germany, Japan, and Denmark in the development and operations. After five years of development it was launched from Kennedy Space Center on 20 November 2004. Full normal operations commenced on 5 April 2005.

Swift catches about two GRBs in a week, and carries out about three ToO observations a day.

\subsubsection{Fermi - A High Energy Observatory}

Fermi has two primary high-energy detectors: the Large Area Telescope (LAT) which operates between $20 \mathrm{MeV}$ and $\gtrsim 300 \mathrm{GeV}$ (Atwood et al. 2009) and the Gamma-Ray Burst Monitor (GBM) which operates between $8 \mathrm{keV}$ and $40 \mathrm{MeV}$ (Meegan et al. 2009). The LAT is a $3000 \mathrm{~kg}$ instrument consisting of $77 \mathrm{~m}^{2}$ of silicon detectors. It is a pair conversion telescope with silicon detector tracker and cesium iodide calorimeter. The LAT scans the full sky every $3 \mathrm{hr}$; all data are made public immediately. The GBM is a scintillator-based instrument with 12 sodium iodide detectors and two bismuth germanate detectors. Fermi was launched into low-Earth orbit in June 2008.

\subsection{Follow-up Telescopes}

Here are descriptions of some of the rapid-response optical telescopes that perform follow-up observations of Swift GRBs and transients.

\subsubsection{RAPTOR (Rapid Telescopes for Optical Response)}

RAPTOR is a suite of telescopes designed to detect and track transient objects, including gamma-ray bursts. It was built by a team at Los Alamos led by Tom Vestrand and consists of six robotic observatories, designated RAPTOR A, B, S, P, K, and T, all located near Los Alamos. The individual telescopes are controlled by a central computer system. The older RAPTOR telescopes, RAPTORs A, B, S, and P each include both a wide-field telescope and a narrow-field telescope. All six are mounted on platforms capable of swiveling to any sky location in $<3 \mathrm{~s}$. Each wide-field telescope has a nominal $38^{\circ} \times 38^{\circ} \mathrm{FOV}$; thus the sky can be covered in eleven pointings. Each telescope is two telescopes, enabling stereoscopic vision. Each telescope also has zoom capability. RAPTOR takes two consecutive $30 \mathrm{~s}$ exposures through its two wide-field telescopes and analyzes the resulting digital images while in monitoring mode. If it sees an interesting event, RAPTOR zooms in for a closer look with the two narrow-field telescopes.

The newest telescopes are RAPTOR-K and RAPTOR-T, which began operation in 2008. RAPTOR-K has 16 lenses and can view the entire sky every $5 \mathrm{~min}$. RAPTOR-T ( $\mathrm{T}=$ technicolor) has four co-aligned $0.4 \mathrm{~m}$ telescopes with four filters. It can therefore provide time-resolved photometry during a GRB, and thereby constrain its distance as well as the GRB's dynamics and circumstellar environment.

RAPTOR participates in the Los Alamos Thinking Telescope Project, an effort to develop hardware and software with the goal of managing logistics of transient object detection. In addition, RAPTOR led to SkyDOT, the Sky Database for Objects in Time-Domain. 
The scientific highlight for RAPTOR has been GRB130427A which was seen peaking at $7^{\text {th }}$ mag (Vestrand et al. 2014). At a redshift of $z=0.34$, it had the largest GRB fluence ever recorded. The burst was accompanied by SN 2013cq (Xu et al. 2013). In contrast to other GRB/SNe with typical $E_{\text {iso }}$ values of $\sim 10^{48} \mathrm{erg}, \mathrm{GRB} 130427 \mathrm{~A}$ had $E_{\text {iso }} \simeq 10^{52} \mathrm{erg}$. A detailed examination of the light curves in different bands allows one to constrain the forward and reverse shocks (Figure 1, from Vestrand et al. 2014).

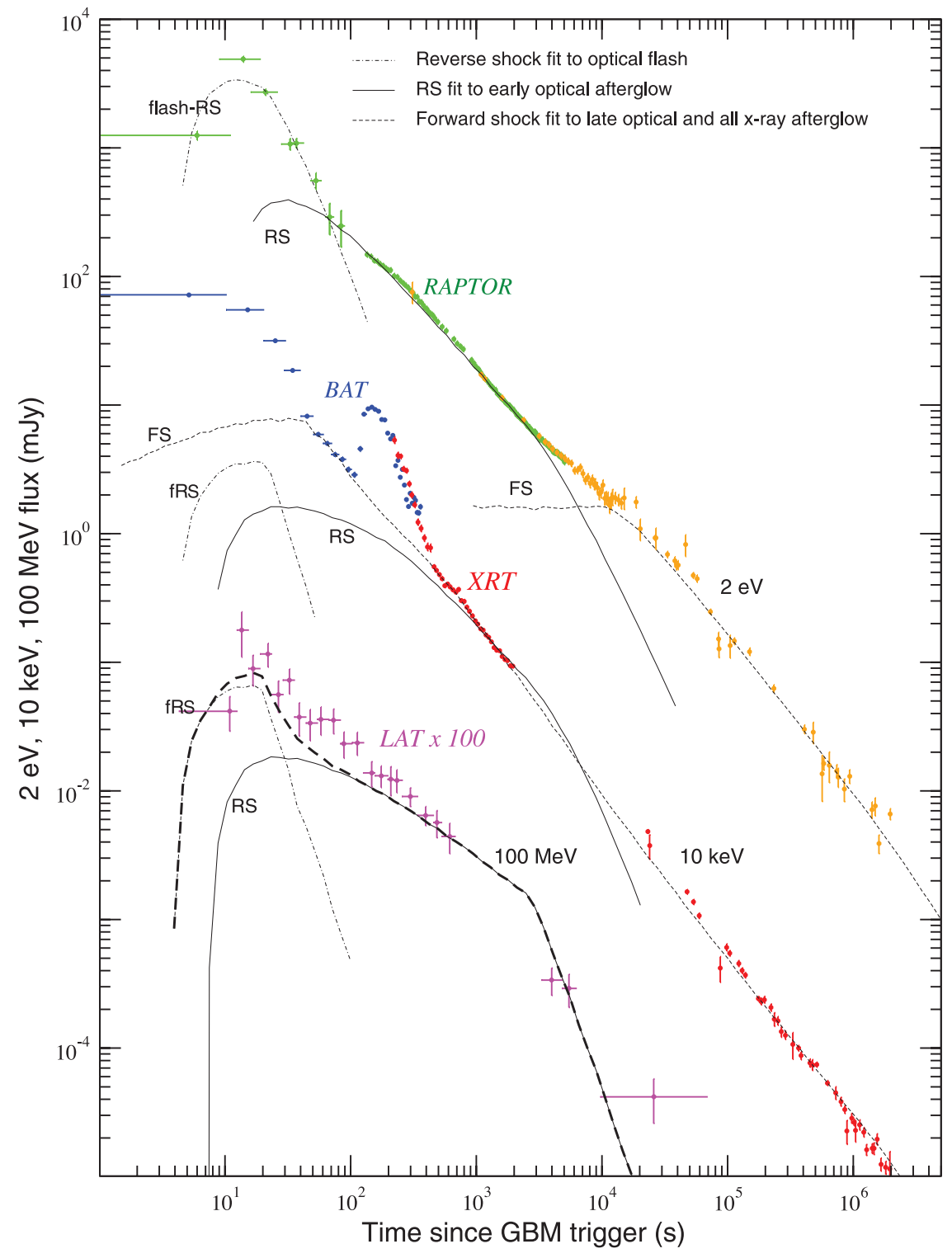

Figure 1: Best-fit with a reverse/forward shock model to the optical light curve of GRB afterglow 130427A (Vestrand et al. 2014). Three episodes of ejecta and energy injection are needed to explain the optical flash, the early optical emission (up to few kiloseconds), and the late optical emission (after a few kiloseconds). 


\subsubsection{Watcher Robotic Telescope}

Watcher is located at Boyden Observatory in South Africa; it saw first light in 2006. Its primary scientific objective is to study the optical prompt and early afterglow emission from GRBs. Watcher consists of a $0.4 \mathrm{~m} \mathrm{f} / 14.25$ Cassegrain primary mirror. It has an Andor EMCCD camera and Optec filter-wheel with BVRI filters. The software runs on two Linux machines, and the entire system is controlled by the open source RTS2 software package.

A sequence of images of a given source is turned into a calibrated light curve via the walapi pipeline which determines the statistical zero-point for each image using all stars in the field. This is being replaced by a faster Python watcher-pipeline that uses several of the most suitable secondary standards within an image for calibration. The APASS catalogue is now used for reference brightnesses, along with the USNO-B1 catalogue. Parameters used in the determination of the target's magnitude are adjusted in real-time based on current seeing, image quality, and the nature of the source, i.e., whether it is a known bright source or a faint GRB afterglow at the limit of detectability.

\subsubsection{Rapid Eye Mount telescope (REM)}

REM is an autonomous telescope at La Silla Observatory whose objective is to catch GRB afterglows. It is triggered automatically by GCN alerts from high-energy satellites such as Swift and rapidly slews to the localization. It is operated by the Italian National Institute for Astrophysics (INAF).

It was built in 2002-2003 and saw first light in September 25, 2003. The telescope is a $0.6 \mathrm{~m}$ Ritchey-Chrétien reflector with $1.16^{\prime \prime}$ resolution, a $9^{\prime} \times 9^{\prime}$ FOV, and an $\mathrm{f} / 2.2$ focal length of $1.32 \mathrm{~m}$. It has $\lambda=450-2300 \mathrm{~nm}$ wavelength range. Its small size facilitates a fast slew rate $\sim 10^{\circ} \mathrm{s}^{-1}$.

The telescope has REMIR, an imaging IR camera, and ROSS, a visible imager and a slitless spectrograph. A dichroic located before the telescope focus enables both cameras to observe the same FOV at the same time. REMIR has $z^{\prime}, J, H$, and $K^{\prime}$ filters; ROSS has standard $V, R, I$ filters and a slitless Amici prism.

The observing procedure is completely robotic and the nightly schedule is optimized for the observation of scheduled targets but it is immediately overdriven by GRB (or other) alerts. Typically REM can observe the new target after 30 seconds from notification.

A wide FOV camera parallel to the REM telescope, TORTORA (Telescopio Ottimizzato per la Ricerca dei Transienti Ottici RApidi), was installed in 2006. TORTORA has a $0.12 \mathrm{~m}$ objective and $24^{\circ} \times 32^{\circ} \mathrm{FOV}$. Its optimized time resolution is $\sim 0.1 \mathrm{~s}$.

\subsubsection{Pi of the Sky}

Pi of the Sky is a system of wide FOV automated telescopes which operates autonomously. It monitors a large fraction of the sky to a depth of $12-13$ mag with time resolution $\sim 1-10 \mathrm{~s}$. The system was successfully tested with a prototype at Las Campanas Observatory (Chile) from 2004 to 2009 and moved to San Pedro de Atacama Observatory in March 2011. In October 2010 the first unit of the final Pi of the Sky detector system, with four CCD cameras, was installed at the INTA El Arenosillo Test Centre in Spain. In July 2013 three more units (12 CCD cameras total) were installed together with the first one, on a new platform in INTA, extending sky coverage to about 6000 square degrees (or $\sim 1.8$ steradian), similar (by design) to that of Swift/BAT. 
The standard model for robotic telescope operations is to receive GCN alerts which then relies on rapid repointing to prompt optical afterglow. This method does not allow for a detection at the same time as the $\gamma$-ray emission, or prior to it. The full Pi of the Sky FOV constantly follows the FOV of Swift in real time such that they both "see" the same patch of sky at all times, thereby eliminating any delay time due to repointing based on a Swift GCN alert. Pi of the Sky has four custom designed CCD cameras per mount. Each camera has $f=85 \mathrm{~mm}$ Canon lenses $(f / d=1.2)$ with $20^{\circ} \times 20^{\circ}$ FOV. The limiting magnitude for a single frame is $12^{\text {th }} \mathrm{mag}$; it rises to $13.5^{\text {th }}$ mag for a frame stacked from 20 exposures. Before 2009 all observations were made in white light, except for an IR-cut filter in order to minimize the sky background. Starting in May 2009 one of the cameras has had a Bessel-Johnson R-band filter in order to facilitate absolute calibration. The full suite consists of 16 cameras placed on four specially designed equatorial mounts (4 cameras per mount). The specially designed equatorial mount allows the cameras to operate in two modes. The camera deflecting mechanism allows either (i) all cameras to point at the same object, with a common $20^{\circ} \times 20^{\circ} \mathrm{FOV}$, or (ii) individual cameras to deflect $13^{\circ}$ along the diagonal of the CCD ship, enabling a $38^{\circ} \times 38^{\circ}$ FOV with one mount.

By selecting only high quality measurements, an average photometry uncertainty $\sim 0.018-$ 0.024 mag has been achieved for stars of 7-10 mag. Photometric accuracy is improved further via a dedicated color correction algorithm. When performing observations without any filter (i.e., for most of the data), measurements are normalized to reference stars measured in V.

The scientific highlight of Pi of the Sky thus far has been GRB 080319B. At 6:12:49 UT on March 19th 2008 Pi of the Sky detected a new object, and a few seconds later a Swift GCN alert arrived indicating the discovery of the extremely luminous GRB. At its peak the optical light reached $5.3 \mathrm{mag}$, which was impressive considering it lay at a redshift of 0.937 . The optical data from Pi of the Sky, combined with panchromatic observations from a variety of observatories, allowed detailed constraints to be placed on the physics of GRB 080319B (Racusin et al. 2008, see section 4 below).

\subsubsection{Liverpool Robotic Telescope (LRT)}

Located at the Roque de los Muchachos Observatory on La Palma, the $2 \mathrm{~m}$ Liverpool Telescope is a fully robotic Ritchey-Chrétien telescope. The $\mathrm{f} / 3$ primary mirror has a $0.45 \mathrm{~m}$ central bore and the secondary mirror is $0.62 \mathrm{~m}$ in diameter. The prime focus image scale is $29.1 \mu \mathrm{m} \mathrm{arcsec}{ }^{-1}$; the Cassegrain image scale is $97 \mu \mathrm{m} \operatorname{arcsec}^{-1}$. The autoguider permits closed loop tracking and corrects tracking errors via guide star monitoring. The autoguider has $1024 \times 1024$ active pixels, 0.22 arcsec pixel $^{-1}, 13 \mu \mathrm{m} \times 13 \mu \mathrm{m}$ size, peak quantum efficiency $95 \%$, operating temperature $-40^{\circ} \mathrm{C}$, and pixel readout rate $1 \mathrm{MHz}$. Translational tracking errors are corrected, rotational errors are not.

LRT has several instruments which are relevant for GRB studies:

Optical Wide Field Camera (IO:O): IO:O does the optical imaging. It is a $4096 \times 4112$ pixel e2v CCD 231 detector with $15 \times 15 \mu \mathrm{m}$ pixel size, 0.15 arcsec pixel scale, and $10^{\prime} \times 10^{\prime}$ FOV. IO:O utilizes a 12 position filter wheel with Sloan $\left(u^{\prime}, g^{\prime}, r^{\prime}, i^{\prime}, z^{\prime}\right)$, Bessell $(B$ and $V)$, and narrow-band filters, $\mathrm{H} \alpha$ rest wavelength $656.28 \mathrm{~nm}$, and redshifted to $663.4,670.5,675.5$, and $682.2 \mathrm{~nm}$.

Near-IR Camera (IO:I): IO:I does the NIR imaging. It consists of a $2048 \times 2048$ pixel Hawaii $2 \mathrm{RG}$ detector with $18 \times 18 \mu \mathrm{m}$ pixel size, 0.18 arcsec pixel scale and $6^{\prime} .3 \times 6^{\prime} .3 \mathrm{FOV}$. IO:I 
utilizes a fixed $\mathrm{H}$ filter.

SPectrograph for the Rapid Acquisition of Transients (SPRAT): SPRAT is a low resolution spectrograph employing a grism for spectroscopy with a wavelength range $\lambda=400-800 \mathrm{~nm}$ and $7^{\prime} .5 \times 1^{\prime} .9 \mathrm{FOV}$. It has a dispersion $0.46 \mathrm{~nm} / \mathrm{pixel}$, a slit length of $95^{\prime \prime}$, and a slit width of $1.8^{\prime \prime}$, giving a resolution of $1.8 \mathrm{~nm}$ (4 pixels), or $R=\lambda / \delta \lambda=350$.

SkyCam: The system consists of three cameras: SkyCam-A, an all sky camera on a fixed mount providing horizon-to-horizon coverage to $\sim 6^{\text {th }}$ mag; SkyCam-T, a medium FOV camera with a roughly $9^{\circ}$ FOV sensitive to $\sim 13-14^{\text {th }}$ mag; SkyCam-Z, a zoomed FOV camera with a $\sim 1^{\circ}$ FOV sensitive to $\sim 18^{\text {th }}$ mag.

The telescope's robotic control system (RCS) accepts observation requests from professional astronomers $24 \mathrm{hrs}$ a day. The RCS additionally can automatically interrupt scheduled observations to observe higher priority transients, such as gamma-ray bursts.

The LRT is one of the largest robotic telescopes. It was built by Telescope Technologies Ltd, a company set up by Liverpool John Moores University, which also owns LRT. It is operated by the Astrophysics Research Institute.

\subsubsection{Reionization and Transients InfraRed (RATIR) camera}

The RATIR camera is a 6 color imaging system $(i, r, z, Y, J$, and $H)$ on the $1.5 \mathrm{~m}$ Johnson telescope at San Pedro Mártir Observatory in Mexico. The telescope is automated with RATIR as the science imager. It is dedicated to the follow-up and identification of high $-z$ GRBs. It can typically observe within $\sim 30 \mathrm{~s}$ of a GCN alert. The nominal pointing error is $\sim 15^{\prime \prime}$ RMS. With a sufficient density of bright stars, this can approach $\sim 1^{\prime \prime}$ RMS. Offset precision is $\sim 2^{\prime \prime}$. Median image quality in $\operatorname{irz} Y J H$ is $\sim 1^{\prime \prime}$.

Three dichroics divide the light from the telescope into four channels, $\mathrm{C} 0$ through $\mathrm{C} 3$, all with a $2048 \times 2028$ pixel format. The first two, C0 and C1, are Fairchild 3041 CCD detectors with $5^{\prime} .4 \times 5^{\prime} .4 \mathrm{FOV}$ and $0.32^{\prime \prime}$ pixel size. For filters, C0 uses SDSS ugr plus seven others, while C1 uses fixed SDSS $i$. Detectors C2 and C3 are HAWAII-2RG detectors with $10^{\prime} \times 10^{\prime}$ FOV and $0.3^{\prime \prime}$ pixel size. C2 uses fixed WFCAM $Z$ and $Y$, while $\mathrm{C} 3$ uses fixed MKO $J$ and $H$.

\subsubsection{VLT X-shooter}

The VLT is operated by ESO at Paranal Observatory, Chile. It consists of four individual telescopes. They are usually used separately but can be employed in unison to obtain high angular resolution. Each telescope is of Ritchey-Chrétien Cassegrain design with a 22 ton, $8.2 \mathrm{~m}$ Zerodur primary mirror, and a $1.1 \mathrm{~m}$ Be secondary mirror. A flat tertiary mirror diverts the light to one of two instruments at the f/15 Nasmyth foci on either side, or tilts aside to allow light through the primary mirror central hole to a third instrument at the Cassegrain focus. Additional mirrors can send the light via tunnels to the central VLTI beam-combiners. The maximum FOV at Nasmyth foci is around 27 arcminutes diameter.

The wavelength range is $\lambda=300 \mathrm{~nm}$ to $20 \mu \mathrm{m}$. Each telescope weighs $\sim 350$ tons, and has active optics: 150 supports on the back of each primary control the shape of the $0.177 \mathrm{~m}$ thick mirror via actuators controlled by computers. 
The X-Shooter is the first second-generation instrument on the VLT, a very wide-band (UV to NIR) single-object spectrometer designed to explore the properties of rare, unusual or unidentified sources, such as GRBs.
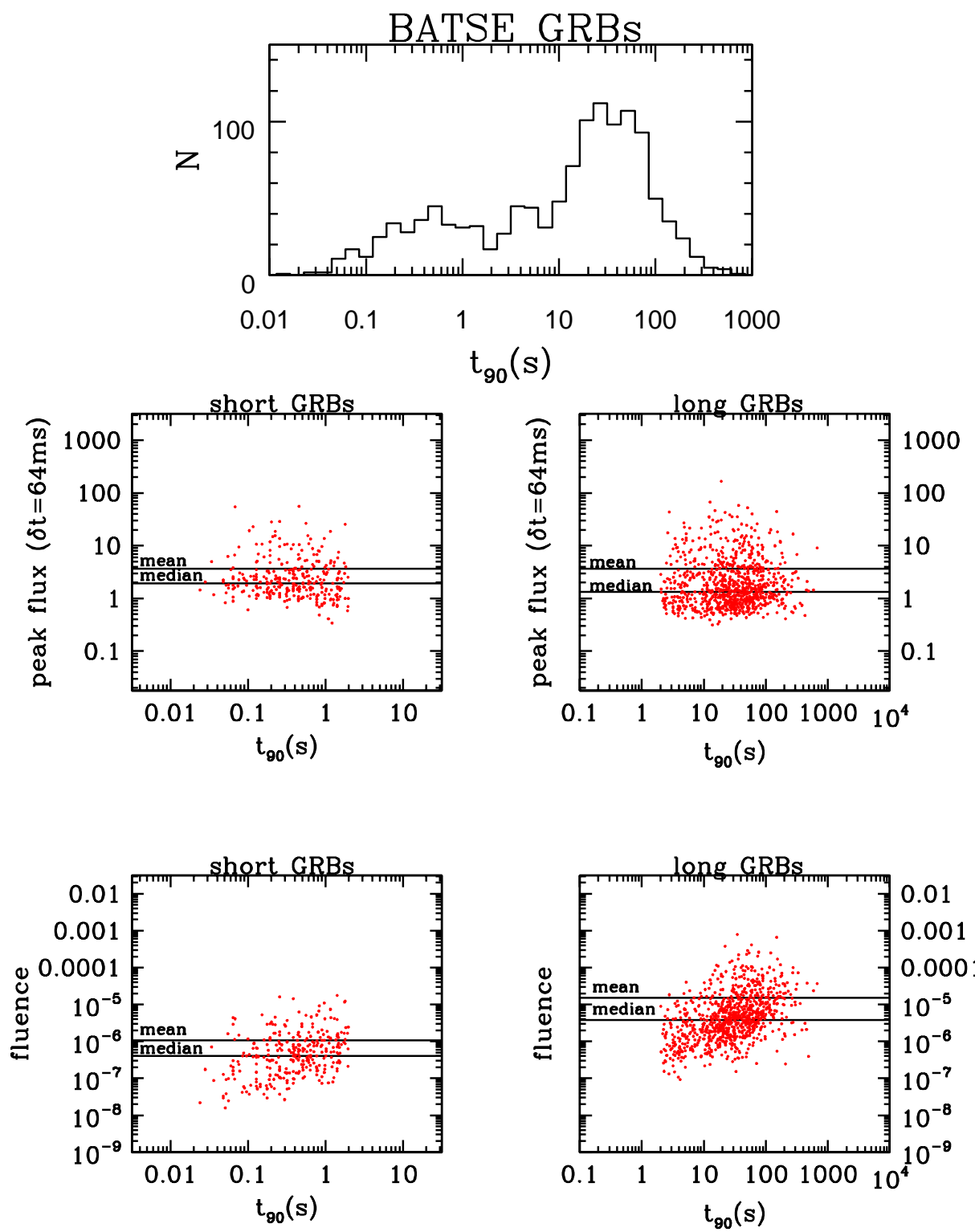

Figure 2: Properties of 1234 BATSE GRBs. 


\section{Properties of GRBs}

Gamma-ray bursts (GRBs) are intense flashes of radiation produced at cosmological distances $z \simeq 2$. GRBs come in two primary flavors, long and short, with the dividing point being roughly $2 \mathrm{~s}$ (Kouveliotou et al. 1993). Figure 2 shows a compendium of 1234 BATSE GRBs. A further division can be made spectrally according to their hardness ratio (i.e., ratio of high to low energies). The redshift range is from about 0.2 to 2 for short GRBs (sGRBs), with a mean of about 0.4. For long GRBs (lGRBs) the range is between about 0.009 and 8.2, with a mean of about 2.3. The typical energy release is $\sim 10^{49}-10^{50} \mathrm{erg}$ for sGRBs and $\sim 10^{50}-10^{51}$ erg for lGRBs. These ranges are based on observed isotropic-equivalent energies of $\sim 10^{51} \mathrm{erg}$ for sGRBs and $\sim 10^{53} \mathrm{erg}$ for lGRBs, and estimates for jet beaming for each class, $\theta_{\mathrm{j}} \sim 5^{\circ}$ for lGRBs and $\theta_{\mathrm{j}} \sim 5-15^{\circ}$ for sGRBs (Burrows et al. 2006, Grupe et al. 2006, Fong et al. 2012). Beaming angles for sGRBs are still highly uncertain. The $L_{X} / E_{\gamma-\text { iso }}$ values at $11 \mathrm{hr}$ post-GRB are similar between lGRBs and sGRBs (Gehrels et al. 2008). The sGRBs have weaker X-ray afterglows, a mean value of $\sim 7 \times 10^{-10} \mathrm{erg}$ $\mathrm{cm}^{-2} \mathrm{~s}^{-1}$ versus $\sim 3 \times 10^{-9} \mathrm{erg} \mathrm{cm}^{-2} \mathrm{~s}^{-1}$ for lGRBs. Although many of the details are uncertain, the two mechanisms are thought to be collapsars for lGRBs and merging neutron stars for sGRBs. LGRBs are intrinsically very bright, the brightest explosions in the Universe. The highest redshift lGRBs were far above detector threshold. SGRBs, by contrast, constitute a flux-limited sample, and are seen primarily nearby, $z \lesssim 1$.

\section{Short GRBs and Kilonovae}

Various groups have explored the supernova-like transient powered by this radioactive decay of material ejected from the NS (Eichler et al. 1989; Li \& Paczyński 1998; Kulkarni 2005; Metzger, Piro, Quataert 2008; Metzger et al. 2010; Metzger \& Berger 2012). The resultant "kilonova" (dimmer than a supernova and brighter than a nova) would produce relatively isotropic optical/NIR emission after a NS-NS/NS-BH merger. While SNIa light curves are powered primarily by decay of ${ }^{56} \mathrm{Ni}$, the ejecta from a disrupted NS is neutron rich and yields little Ni. Much heavier radioactive elements form via rapid neutron capture ( $r$-process) nucleosynthesis following the decompression of the ejecta from nuclear densities. These newly synthesized elements undergo nuclear fission, $\alpha$ and $\beta$ decays on much longer time-scales. The resulting energy release can power detectable thermal emission once the ejecta expands sufficiently that photons can escape.

Kasen et al. (2013) argue that the opacity of the expanding $r$-process material is dominated by bound-bound transitions from those ions with the most complex valence electron structure, i.e., the lanthanides. They compute atomic structure models for a few representative ions in order to calculate the radiative transition rates for tens of millions of lines, and find that resulting $r$-process opacities are orders of magnitude larger than that of ordinary (e.g., iron-rich) supernova ejecta. The resultant light curves should be longer, dimmer, and redder than previously thought (Figure 3 ). The spectra have broad absorption features and peak in the IR $(\sim 1 \mu \mathrm{m})$.

Specific predictions for kilonova light curves are dependent on uncertainties such as the type of ejecta (dynamical and/or disk outflows), ejecta masses, and velocities. Recent time dependent calculations find ejecta masses in the range $\sim 10^{-3}-10^{-1} M_{\odot}$ and velocities $\sim 0.1-0.3 c$ (e.g., Foucart et al. 2011; East \& Pretorius 2012; Piran et al. 2013; Kyutoku, Ioka, \& Shibata 2013; Hotokezaka 


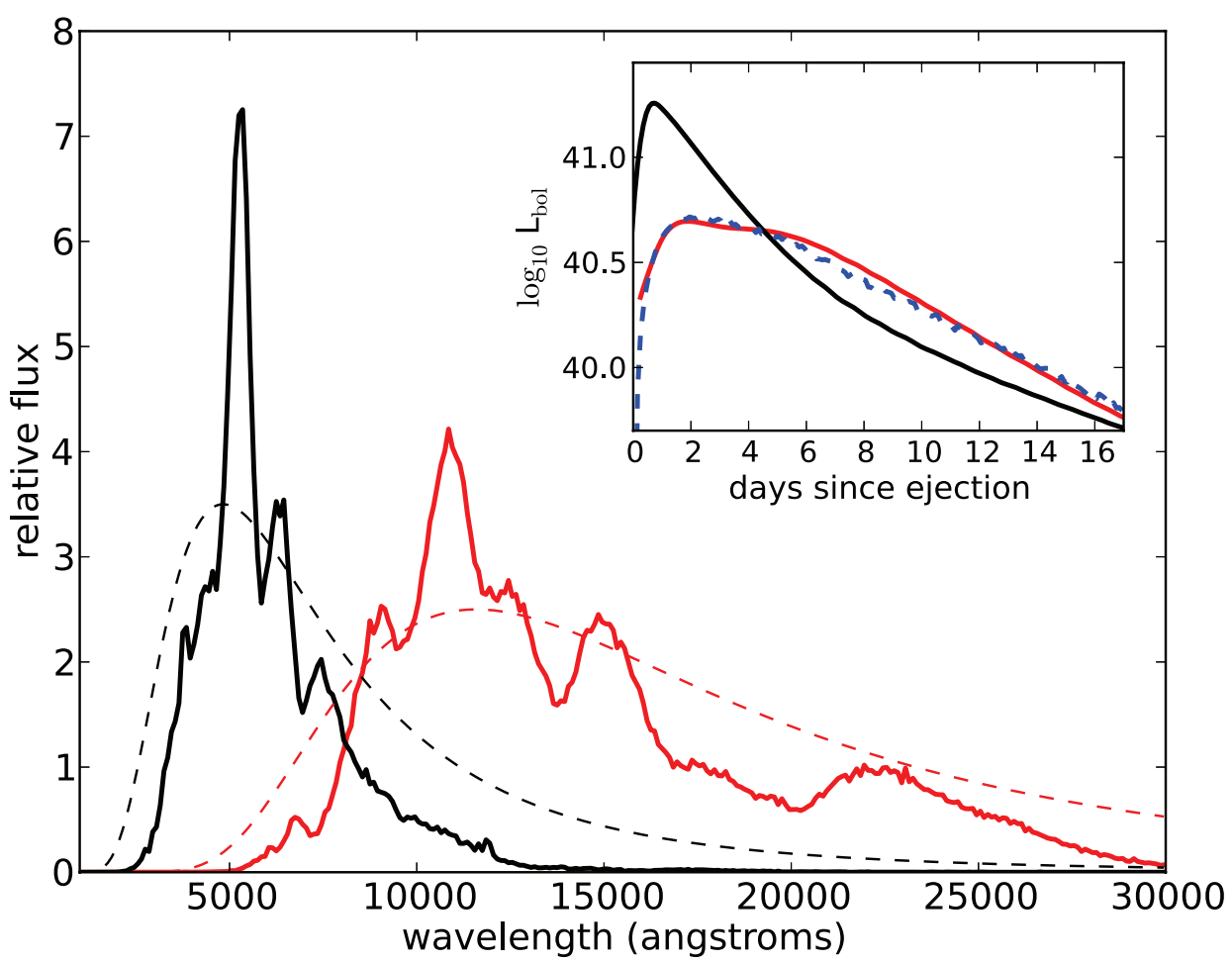

Figure 3: Synthetic spectra ( $2.5 \mathrm{~d}$ after mass ejection) of the $r$-process SN model (Kasen, Badnell, \& Barnes 2013), i.e., a "kilonova", calculated using Kurucz iron group opacities (black line) or Autostructurederived $r$-process opacities (red line). For comparison, also shown are blackbody curves with $T=6000 \mathrm{~K}$ (black dashed) and $T=2500 \mathrm{~K}$ (red dashed). The inset shows the corresponding bolometric light curves assuming iron (black) or $r$-process (red) opacities. Also shown is a light curve calculated with a gray opacity of $\kappa=10 \mathrm{~cm}^{2} \mathrm{~g}^{-1}$ (blue dashed line).

et al. 2013; Fernández \& Metzger 2013; Sekiguchi et al. 2015; Foucart et al. 2015; Fernández et al. 2015). Assuming iron-rich supernova ejecta, Metzger \& Berger (2012) predict peak optical luminosities $\sim 10^{41}-10^{42} \mathrm{erg} \mathrm{s}^{-1}$ and concomitant $M_{R}$ values -14 to -17 . For $M_{\text {eject }}=10^{-2} M_{\odot}$ and $v_{\text {ejecta }}=0.1 c$, Barnes \& Kasen (2013) calculate a peak absolute magnitude in the near-IR $(\lambda \simeq 1.7 \mu \mathrm{m})$ of $M_{H}=-15.5$, in good agreement with Tanaka \& Hotokezaka (2013), Tanaka et al. (2014), and Grossman et al. (2014).

A consideration of the structure of valence shell electrons in different blocks of elements in the periodic table reveals why the lanthanides provide the lion's share of the opacity (Barnes \& Kasen 2013). The lanthanides and actinides reside in the f-block of the periodic table, i.e., those characterized by orbital angular momentum $l=3$. The periodic table is divided into four blocks depending on the $l$ value of an atom's valence shell: s-block $(l=0)$, p-block $(l=1)$, d-block $(l=2)$, and f-block $(l=3)$. In a given atom the orbital angular momentum quantum number $l$ can run from 0 to $n-1$, where $n$ is the principal quantum number, the magnetic quantum number $m_{l}$ can run from $-l$ to $+l$, including 0 , and the electron spin quantum number $m_{s}$ can be $-1 / 2$ or $+1 / 2$. Therefore the number of potential states $g$ in which an electron can appear, for given $l$, is $g=2(2 l+1)$. Once a specific electron is assigned a state, the Pauli exclusion principle posits that subsequent valence 


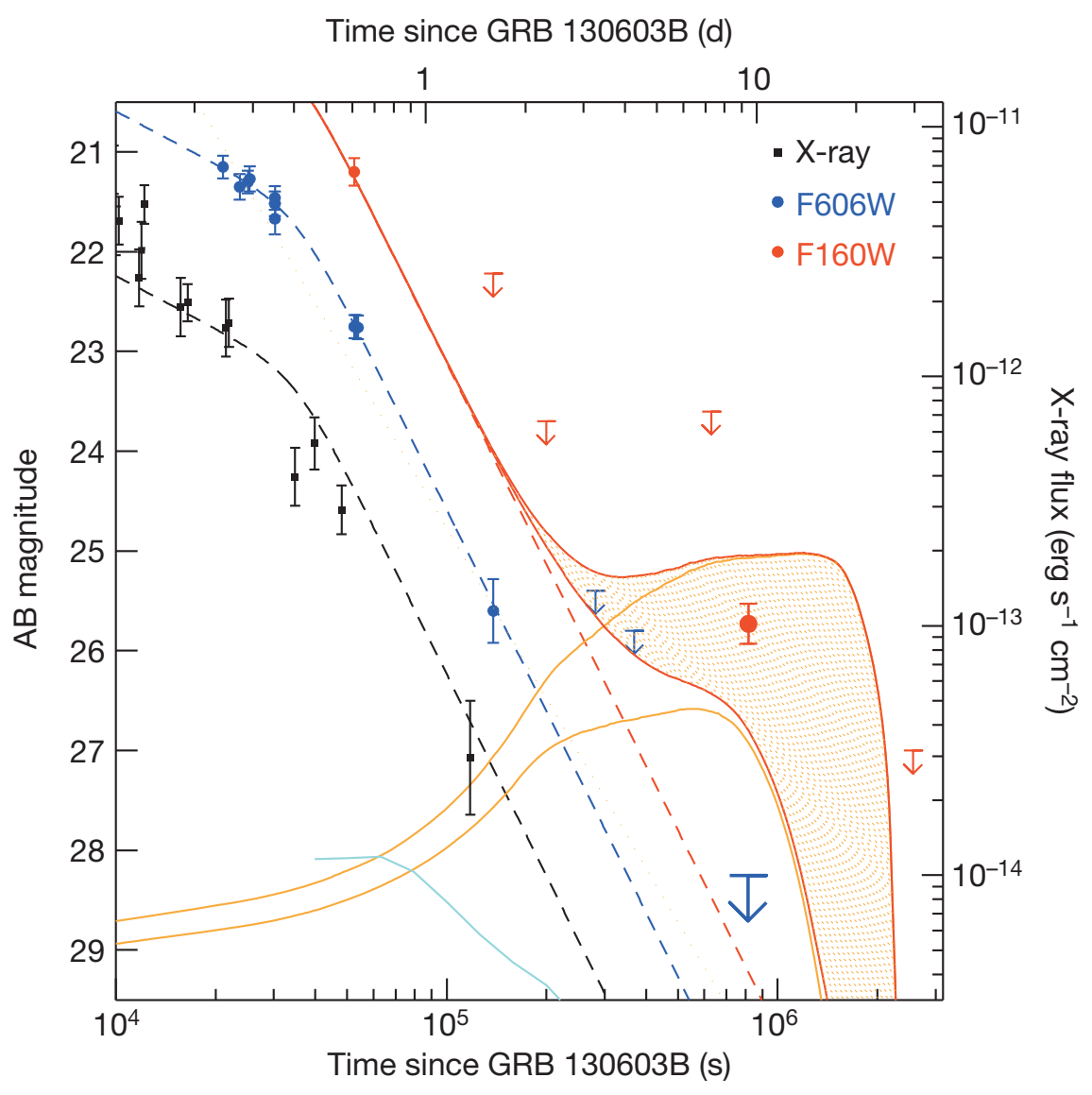

Figure 4: Optical, NIR and X-ray light curves of GRB 130603B (Tanvir et al. 2013). Left axis, optical and NIR; right axis, X-ray. Optical data ( $g, r$ and $i$ ) have been interpolated to the HST F606W band and NIR data have been interpolated to the F160W band using an average spectral energy distribution at $\sim 0.6 \mathrm{~d}$ Absence of late-time optical emission places a limit on any separate ${ }^{56} \mathrm{Ni}$-driven decay component. The $0.3-10 \mathrm{keV}$ Swift/XRT data (Evans et al. 2007, 2009) are also consistent with breaking to a similarly steep decay (the dashed black line shows the optical light curve simply rescaled to match the X-ray points in this time frame), although the source had dropped below Swift/XRT sensitivity by $\sim 48 \mathrm{hr}$ (our frame). This excess NIR flux corresponds to absolute magnitude $M(J)_{\mathrm{AB}} \approx-15.35$ at $\sim 7 \mathrm{~d}$ (source frame). Model lines (Barnes \& Kasen 2013; orange curves) correspond to ejected masses of $0.01 M_{\odot}$ (lower curve) and $0.1 M_{\odot}$ (upper curve), and these are added to the afterglow decay curves to produce predictions for the total NIR emission, shown as solid red curves. The cyan curve shows that even the brightest predicted $r$-process kilonova optical emission is negligible.

electrons cannot occupy that same quantum configuration $\left(n, l, m_{l}, m_{s}\right)$. Therefore the number of ways in which $v$ valence electrons can be put into $g$ distinct states in an orbital is determined 
by the choose function ${ }_{g} C_{v} \equiv g ! /[(v !(g-v) !]$. This gives the number of potential distinct energy configurations in an atom arising from its valence electrons. Since absorption lines are due to transitions between distinct states, the number of lines varies approximately as $C^{2}$ (some transitions being forbidden).

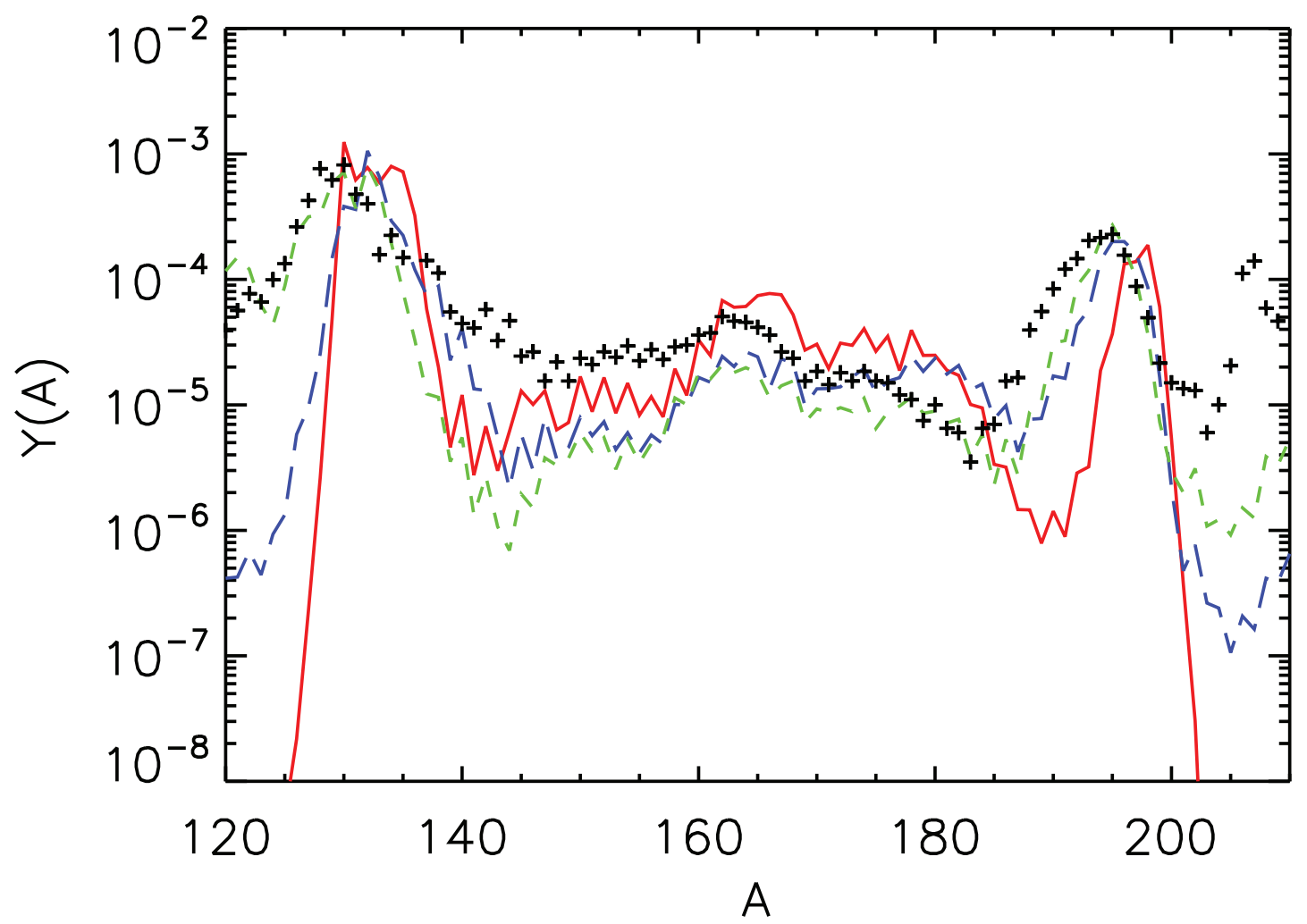

Figure 5: Abundances versus mass number for the three baseline r-process simulations (Surman et al. 2014): a parameterized wind hot r-process (solid red line), supernova neutrino-driven wind cold r-process (long-dashed blue line), and a neutron star merger r-process (short-dashed green line). The scaled solar abundances (crosses) are shown for comparison (Sneden et al. 2008).

Let us compare the number of lines arising from valence electrons in Fe (iron) and Pm (promethium), for example. Fe is a d-block element, hence $l=2$, and its electron configuration is [Ar] $3 \mathrm{~d}^{6} 4 \mathrm{~s}^{2}$; there are $v=6$ electrons in the valence shell. Thus $g(\mathrm{Fe})=2(2 * 2+1)=10$ and $C(\mathrm{Fe})={ }_{10} C_{6}=210$ distinct energy states, giving rise to $\sim 210^{2} \simeq 40,000$ lines. Being a lanthanide, $\mathrm{Pm}$ is a f-block element, hence $l=3$, and its electron configuration is $[\mathrm{Xe}] 6 \mathrm{~s}^{2} 4 \mathrm{f}^{5}$; there are $v=5$ electrons in the valence shell. Thus $g(\mathrm{Pm})=2(2 * 3+1)=14$ and $C(\mathrm{Pm})={ }_{14} C_{5}=2002$ distinct energy states. Thus the number of potential lines for $\mathrm{Pm}$ is $\sim(2002)^{2}$ or about 4 million - 100 times that of Fe! For ordinary non-r-process stellar material the relative abundances of the lanthanides are so low that this extra absorbing power per atom is not relevant in terms of the overall, effective opacity. However, for r-process material the relative abundances of the synthesized elements are relatively constant with atomic number (Figure 5).

The importance of the lanthanides vis a vis the opacity was not appreciated in the first detailed 
work, by Metzger et al (2010), in which the interval from GRB start time $T_{0}$ to optical peak was too long by a factor of $\sim 3$, and the peak luminosity too high by a factor $\sim 10^{3}$. In addition, current calculations (Fontes et al. 2017) indicate the IR also peaks three times later and is a factor of a hundred fainter than in Metzger et al (2010). Fontes et al. (2017) present the most up to date calculations using the extensive Los Alamos opacity codes. They point out that most atomic calculations neglect lines that are very narrow; this underestimates the total opacity. Broadening these lines while conserving equivalent width increases the opacity substantially. Fontes et al. (2017) discuss two approaches, expansion opacity and opacity broadening. With expansion opacity, one assumes the lines are sparse (i.e., non-overlapping) so that each line can be treated separately and each interaction as a scattering event. This leverages the Sobolev approximation for the velocity gradient, which helps photons diffuse relative to lines. In the opacity broadening approach, one assumes the lines are dense (i.e., overlapping), which means that a photon quickly enters a new line once it leaves the previous one. Fontes et al. (2017) delineate a range of behavior spanning these two assumptions. They also explore fully relativistic versus semi-relativistic methods.

An additional complication in calculating spectra and light curves stems from the uncertain lifetime of the hot merger remnant NS which may exist for $\sim 0.1-0.3 \mathrm{~s}$ before collapse to a $\mathrm{BH}$. During this time the NS winds are subject to high neutrino irradiation which can stop r-process nucleosynthesis before lanthanides are produced (Kasen, Fernández, \& Metzger 2015). This will produce transients more in accord with the original study by Metzger et al (2010). In general, kilonova light curves will be due to an admixture of two components, a brief ( $\sim 2$ d) blue optical transient coming from the outer lanthanide-free ejecta, and a slower $(\sim 10 \mathrm{~d})$ IR transient produced in the inner, lanthanide rich layers. Kasen, Fernández, \& Metzger (2015) find that if the hot merger remnant exists for $\gtrsim 0.3 \mathrm{~s}$, the electron fraction in the wind ejecta will be $\gtrsim 0.25$, preventing lanthanide production and thereby squelching the IR transient.

What are the prospects for observing a kilonova in conjunction with a sGRB? The biggest problem is that, since the kilonova emission is weak, it could usually be masked by the normal afterglow. The only hope is for a sGRB occurring in a very low density interstellar medium. Observational confirmation of such an event would be important given that this mechanism may be the predominant source of stable $r$-process elements in the universe (Freiburghaus, Rosswog, \& Thielemann 1999; Goriely, Bauswein, \& Janka 2011).

GRB 130603B might be the first detected kilonova (Tanvir et al. 2013; Berger, Fong, \& Chornock 2013). It was a sGRB at $z=0.356$ with a duration $\sim 0.2 \mathrm{~s}$ in the BAT. Tanvir et al. (2013) present optical and near-infrared observations that provide strong evidence for an accompanying kilonova (Figure 4). If correct, it would confirm that compact-object mergers are the progenitors of sGRBs and also the sites of significant production of $r$-process elements. It also offers hope that kilonovae provide an alternative, unbeamed electromagnetic signature of the most promising sources for direct detection of gravitational waves.

\section{Swift reverse shock, naked eye GRB}

In the standard fireball model, relativistic shells within a jet propagate away from the central engine and into the surrounding medium, generating a forward shock (FS). A reverse shock (RS) propagates back into the jet. Studies of the GRB afterglow FS/RS emission can potentially provide 
information about the explosion energy, geometry, and structure of the circumburst medium (Sari, Piran, \& Narayan 1998; Chevalier \& Li 2000). The most useful probe of the initial bulk Lorentz factor $\Gamma_{\text {eff }}$ and the ejecta composition is the RS. The combination of large RS speed $v_{\mathrm{RS}} \sim c$ and the finite and limited ejecta length means that the only hope of directly observing the RS is via its early-time emission, basically optical and/or radio detections. To be detectable very bright bursts are needed. The RS emission is expected to produce a synchrotron spectrum similar to the FS, with well-defined RS/FS properties (Sari \& Piran 1999ab; Kobayashi \& Zhang 2003ab; Zou, Wu, \& Dai 2005). There have been detections of hints of an RS-like component in a handful of bursts. A detailed understanding of RS emission requires a careful decomposition of the afterglow spectral energy distribution (SED) into RS and FS components. Since the peak frequencies of the two components are related by a factor of $\Gamma_{\text {eff }}^{2} \gtrsim 10^{4}$, such a decomposition requires multi-wavelength observations spanning several orders of magnitude in frequency (Laskar et al. 2013).

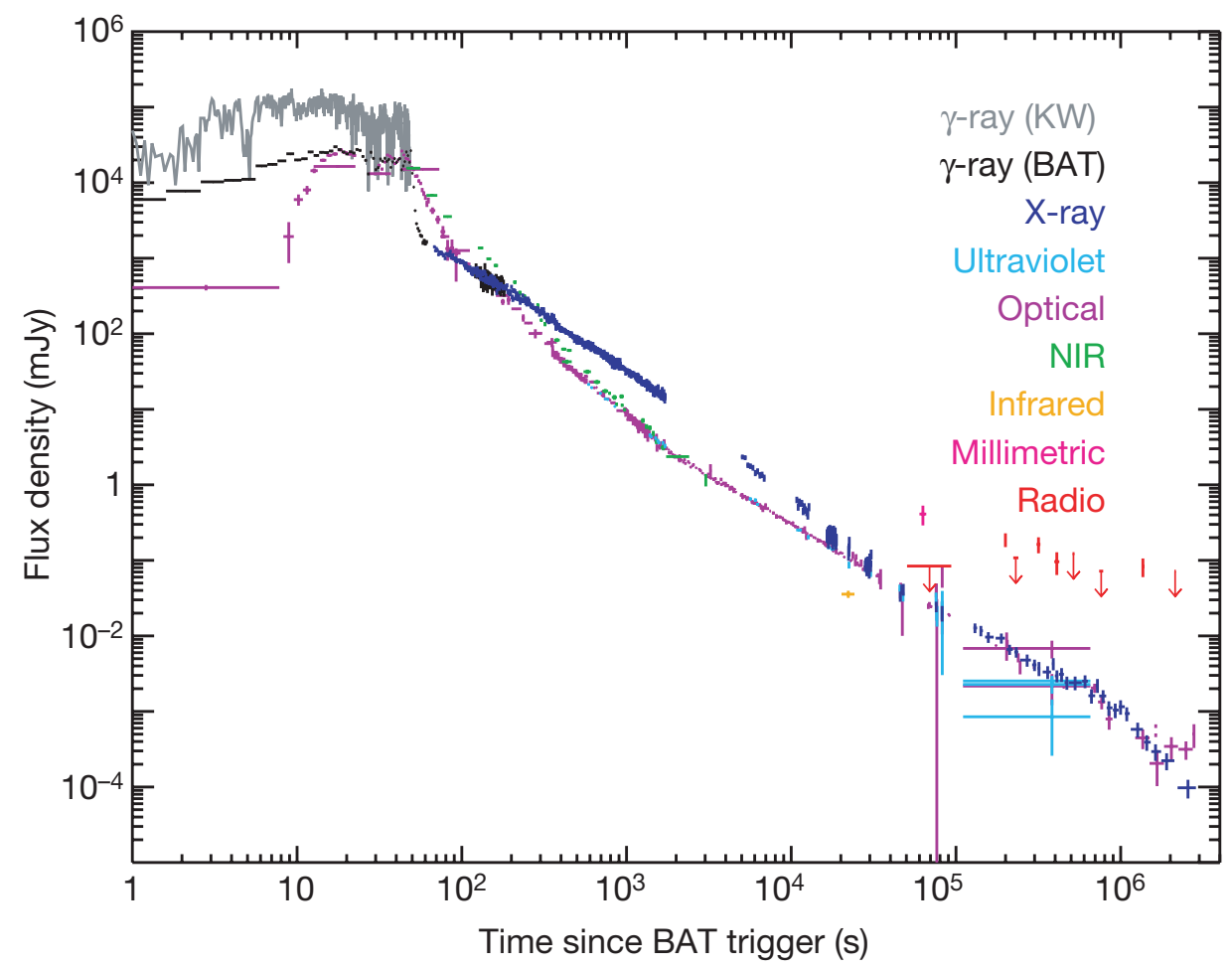

Figure 6: Broadband light curve of the naked eye GRB 080319B, including radio, mm, IR, NIR, optical, UV, X-ray and $\gamma$-ray flux densities (Racusin et al. 2008). The UV, optical and NIR data are normalized to the UVOT $v$-band for $1.5 \mathrm{ks}<(t-T 0)<10 \mathrm{ks}$. Swift/BAT data are extrapolated down into the XRT bandpass $(0.3-10 \mathrm{keV})$ for direct comparison with the XRT data. Combined X-ray and BAT data are scaled up by a factor of 45, and the Konus-Wind data are scaled up by a factor of $10^{4}$ for comparison with the optical flux densities. This figure includes one VLA radio data point (Soderberg, Chandra, \& Frail 2008), and optical data from KAIT, Nickel and Gemini-South (Bloom et al. 2009).

On 19 March 2008 Swift detected the naked eye GRB 080319B (Racusin et al. 2008). It was the brightest optical burst ever observed. If it were $2 \mathrm{kpc}$ from Earth it would have been as bright as the noon sun in the sky. It had a redshift $z=0.937$, a peak visual magnitude 5.3, and a total energy 
in $\gamma$-rays $E_{\text {iso }}=1.3 \times 10^{54} \mathrm{erg}(20 \mathrm{keV}-7 \mathrm{MeV})$. This burst (Figure 6) was observed with a wide variety of instruments spanning the spectrum from radio to $\gamma$-ray. The earliest data at $t<50 \mathrm{~s}$ reveal a common shape for the bright optical and $\gamma$-ray light curves, indicating they arise from the same physical region. The second optical component $(50 \mathrm{~s}<t<800 \mathrm{~s})$ shows the distinct characteristic of a RS, namely, an excess above a time-reversed extrapolation from the later optical power law decay. The final component (at $t>800 \mathrm{~s}$ ) is the afterglow produced as the external FS propagates into the surrounding medium. Previous measurements of GRBs had never revealed all three optical components in the same burst with such clarity. RS emission did not become visible until the prompt emission faded. The high peak luminosity of the optical RS so soon after the end of the $\gamma$-ray emission indicated that the RS was at least mildly relativistic. Furthermore, the GRB outflow could not have been highly magnetized when it crossed the RS or the RS itself would have been suppressed. On the other hand, the presence of strong optical emission accompanying the RS demands some magnetization, therefore an intermediate magnetization seems to be indicated (Racusin et al. 2008).

\section{Tools to Study the High-z Universe}

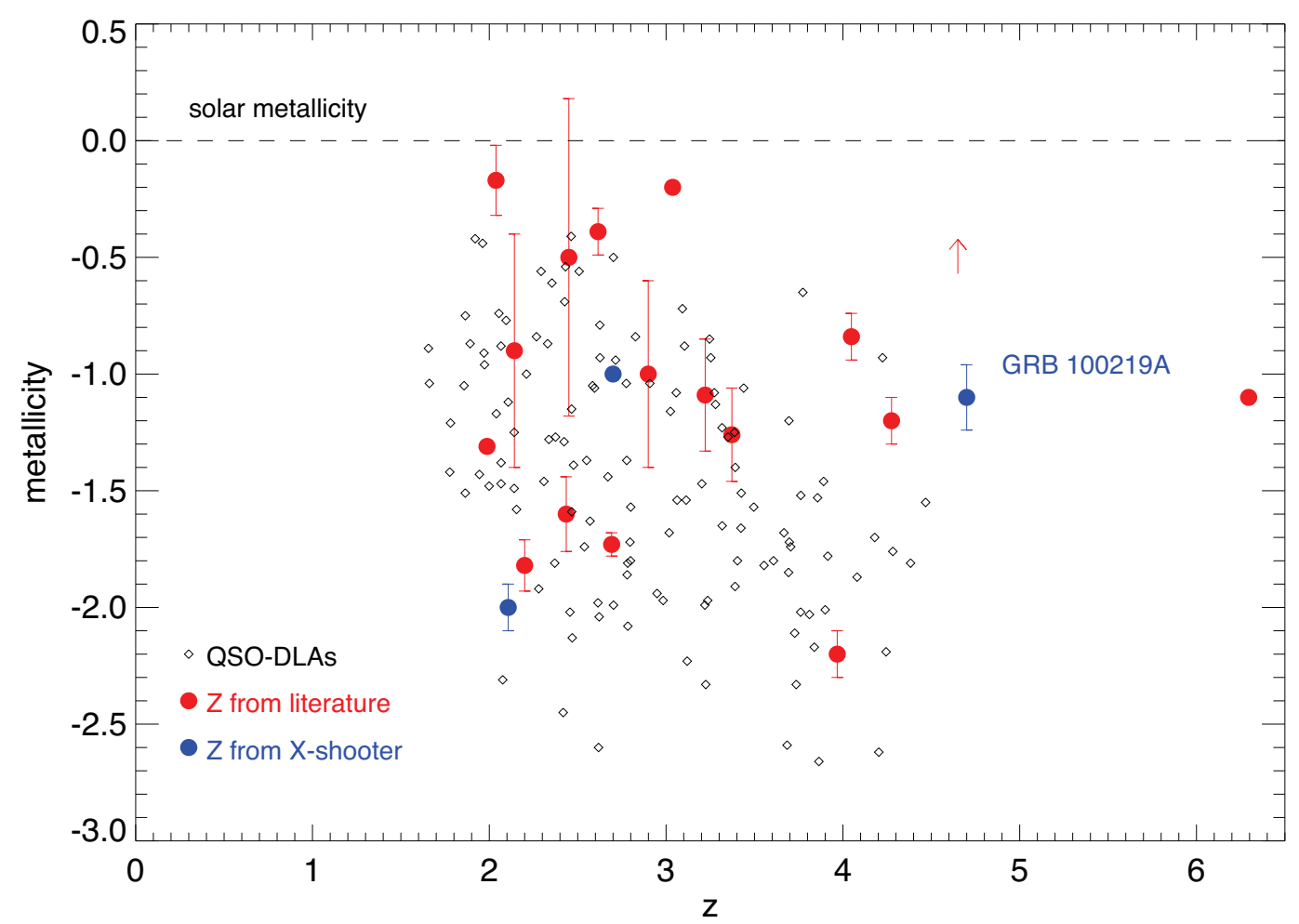

Figure 7: A comparison of metallicities of high $z$ galaxies (red) with QSOs (black) and GRBs (blue), from Thöne et al. (2011). Data are from Prochaska et al. (2003), Fynbo et al. (2006), and Savaglio et al. (2009).

GRBs are incredibly bright. A typical galaxy at a redshift of only $z=3$ is fainter than $m \simeq 27$, whereas the optical component of GRB prompt emission, when seen, can be as high as $m \simeq 10-$ 
15. For GRBs, the current record holder is GRB 090429B at $z \simeq 9.4$ (Cucchiara et al. 2011). Multiwavelength observations of high $z$ GRBs are providing information about the universe at a time when it was only about $4 \%$ of its current age, and shed light on the process of reionization in the early universe (Tanvir et al. 2009; Salvaterra et al. 2009). Strong absorption lines detected in QSO spectra, damped Lyman $-\alpha$ (DLA) systems, originate in galaxies crossing sight lines. A study of the DLA systems associated with optical spectra of GRBs and their hosts has provided detailed information on the metallicity history of the universe, and allowed a comparison of the metallicity history inferred from similar studies involving QSOs. For instance Savaglio (2006) finds that the metallicity for GRBs on average is $\sim 5$ times larger than in QSOs (Figure 7, from Thöne et al. 2011).

GRBs are also being used to determine the star formation rate to high redshift (Figure 8, from Kistler et al. 2009; see also Robertson \& Ellis 2012; Trenti et al. 2015). Corrections need to be made for systematic effects that alter the proportionality between measured GRB rates and inferred star formation rates, such as possible metallicity bias. The star formation rate from GRBs is higher than from other techniques. GRBs provide a more complete measure of star formation from all types of galaxies.

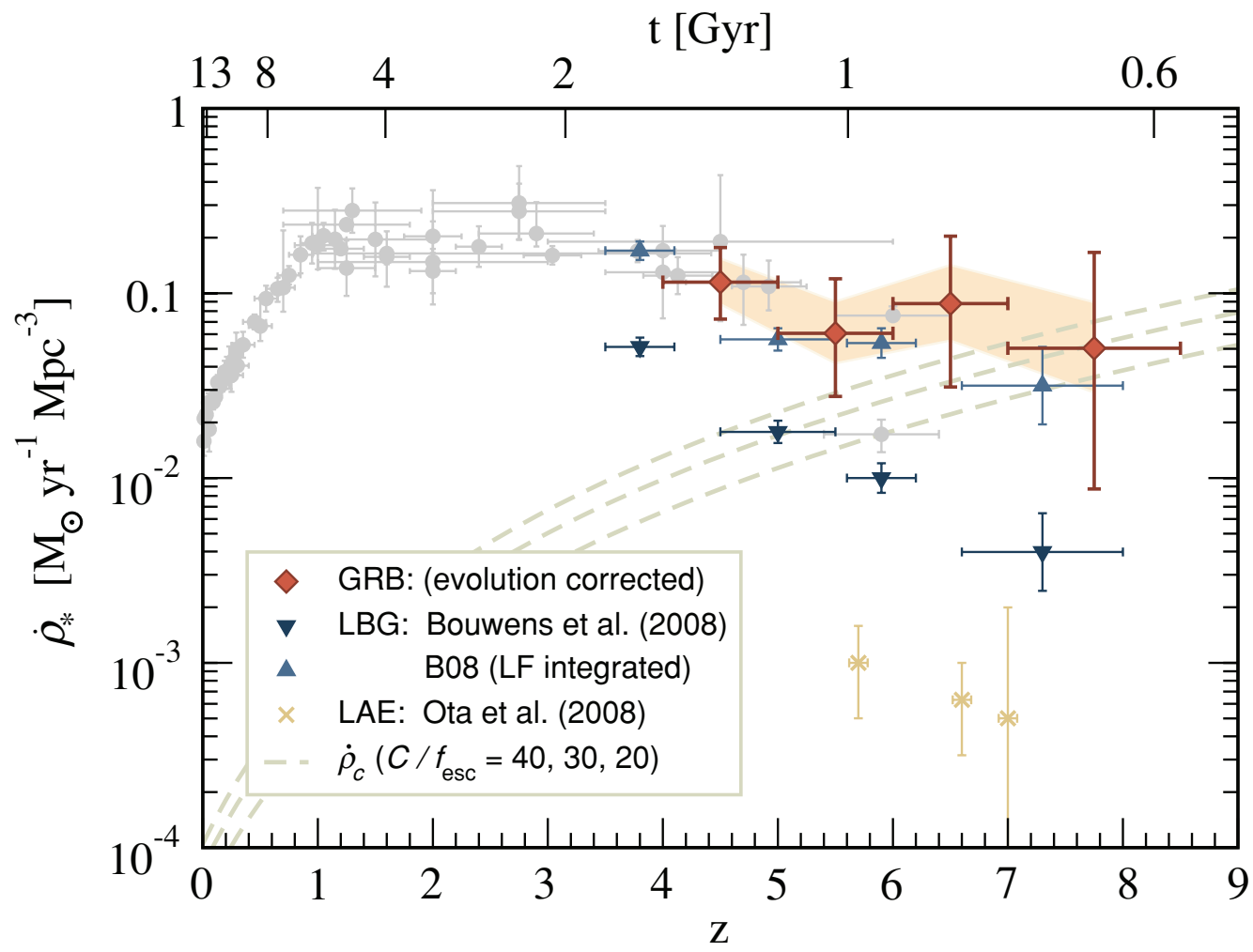

Figure 8: The cosmic star formation rate (SFR) history (Kistler et al. 2009), with data from several sources (Hopkins \& Beacom 2006; Bouwens et al. 2008; Ota et al. 2008). The rates inferred from high $-z$ GRBs are shown as diamonds. The three dashed curves (Madau et al. 1999) give the critical SFR $\dot{\rho}_{c}$ required to balance recombination, for $C / f_{\text {esc }}=40,30$, and 20 (top to bottom), where $C$ is the clumpiness of the intergalactic medium and $f_{\text {esc }}$ the fraction of photons that escape their galaxy. 
Table 1: High $z$ GRBs.

\begin{tabular}{cccccc}
\hline \hline \multicolumn{7}{c}{ Table 2: High Redshift GRBs } \\
\hline \hline$z$ & $t_{\text {look back }}($ Gyr $)$ & GRB & Brightness & \\
\hline 9.4 & 13.1 & $090429 \mathrm{~B}$ & $K=19$ & $@$ & $3 \mathrm{hr}$ \\
8.2 & 13.0 & 090423 & $K=20$ & $@$ & $20 \mathrm{~min}$ \\
$\sim 8$ & 13.0 & $120923 \mathrm{~A}$ & & & \\
7.5 & 13.0 & $100905 \mathrm{~A}$ & $H \simeq 19$ & & \\
6.7 & 12.8 & 080813 & $K=19$ & $@$ & $10 \mathrm{~min}$ \\
6.3 & 12.8 & 050904 & $J=18$ & $@$ & $3 \mathrm{hr}$ \\
6.2 & 12.8 & $120521 \mathrm{C}$ & & & \\
5.9 & 12.7 & $130606 \mathrm{~A}$ & $I=17$ & & \\
5.6 & 12.6 & 060927 & $I=16$ & $@$ & $2 \mathrm{~min}$ \\
5.3 & 12.6 & 050814 & $K=18$ & $@$ & $23 \mathrm{hr}$ \\
5.1 & 12.5 & 060522 & $R=21$ & $@$ & $1.5 \mathrm{hr}$ \\
\hline
\end{tabular}

GRBs, nature's most powerful and luminous explosions, provide us with a means of studying the early Universe. GRBs can trace the early time evolution of star formation, reionization, and metallicity. (Lamb \& Reichart 2000; Lamb 2002; Ciardi \& Loeb 2000; Bromm \& Loeb 2002). At early times GRBs are a hundred to a thousand times brighter than QSOs; they are also expected to be detected beyond $z \approx 10$, while QSOs diminish rapidly beyond $z \approx 3$. In addition GRB afterglows have simple power-law spectra lacking emission lines, and hence are "clean" probes of the intergalactic medium (IGM). Table 1 gives the ten highest-redshift Swift GRBs. About 3\% of Swift GRBs lie at $z>5$, lower than pre-Swift estimates (Bromm \& Loeb 2002). Bromm \& Loeb (2002) also predict that Swift can detect GRBs to $z \gtrsim 8$ - three have been seen to date.

Swift's fast localizations are enabling high $-z$ GRB afterglow spectroscopy. At low resolution a typical host galaxy appears as a damped Ly- $\alpha$ (DLA) system. In addition one sees a wealth of metallic lines which can be used to infer metal abundances. At high resolution the host absorption lines split into an array of fine-structure transitions, allowing an inference of gas densities and of conditions associated with diffuse radiation in the host galaxy (Chen et al. 2005; Berger et al. 2006). Figure 9 presents an optical spectrum for GRB 050505 at $z=4.3$ (Berger et al. 2006). Numerous lines are apparent, including a damped Ly- $\alpha$ feature corresponding to a neutral hydrogen column density of $10^{22} \mathrm{~cm}^{-2}$. The lines imply a density of $100 \mathrm{~cm}^{-3}$ in the source region. Absorption lines observed in infrared spectroscopic observations of GRB 050904 gave a metallicity measurement of 5\% solar (Kawai et al. 2006), the first metallicity determination at such high redshift, demonstrating that the observed evolution in the mass- and luminosity- metallicity relationships from $z=0$ to 2 continues to $z>6$ (Berger et al. 2006).

\section{Short GRB Update}

To date, Swift has detected and positioned to arcsec accuracy 105 sGRBs. The afterglows are weak and fade fast, making them more difficult to study than for lGRBs. Redshift distributions given by Berger (2009) and Fong et al. (2014) must be treated with caution, as there is often a lack of stress on the uncertainties associated with many of the measurements. In particular, often beaming angles or limits on beaming angles are based on two detections and one upper limit. 


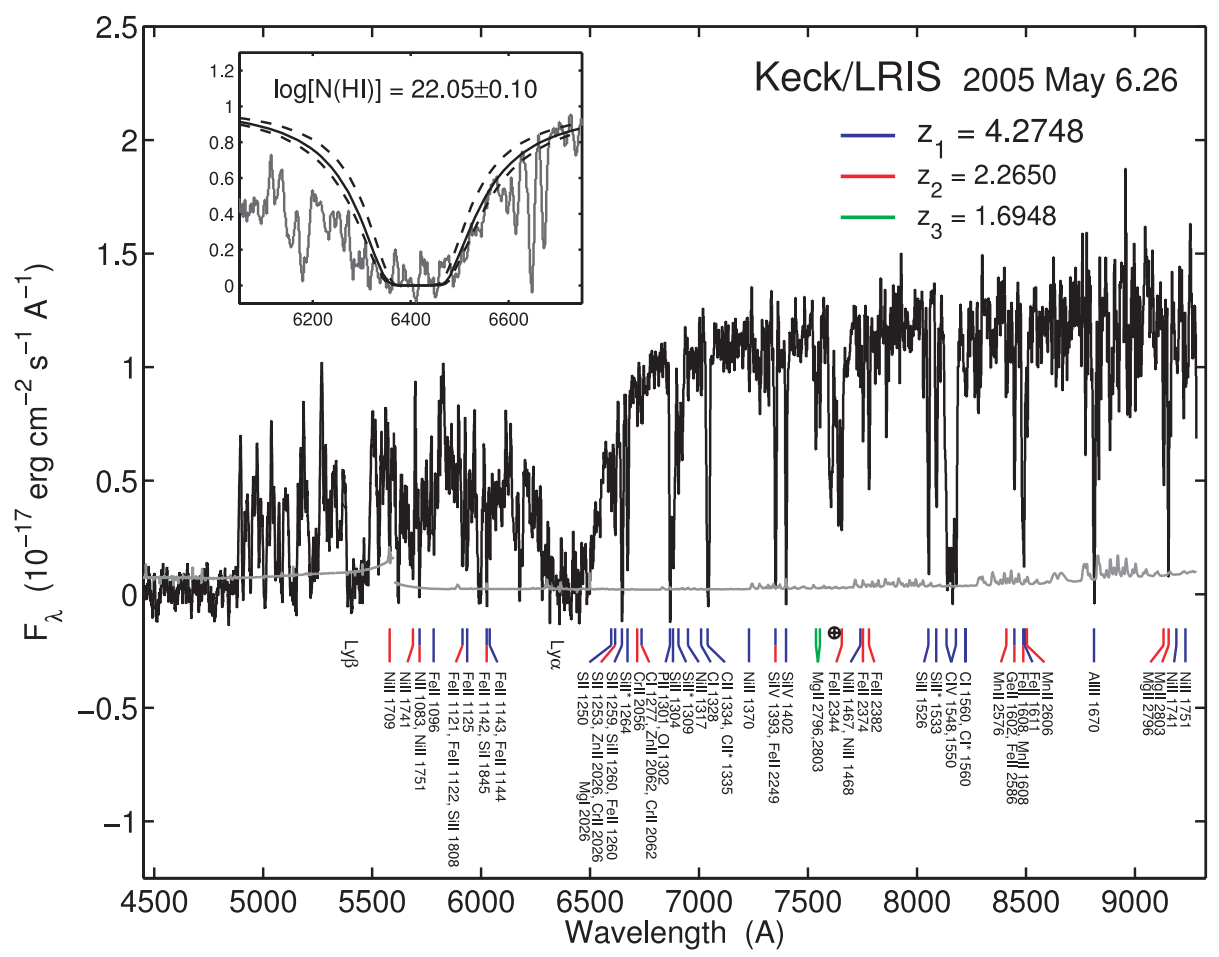

Figure 9: The optical spectrum of GRB 050505 (Berger et al. 2006). Lines are seen from the host galaxy at $z=4.275$ and two foreground absorbers. Inset shows a zoom-in of the Ly $-\alpha$ absorption - solid line shows best fit $\log N(\mathrm{H} \mathrm{I})=22.05$ and dashed lines indicate \pm 1 dex uncertainty.

Figure 10 highlights the advantage in having prompt X-ray emission associated with sGRBs, and also the problems which arise in those lacking a detection of prompt emission. Consider GRB150101B. This was a ground-detected sGRB with very late follow-up. In such cases, it is often problematic to be sure of an association of an X-ray detection with the GRB. For instance, the red points indicate XRT emission which turned out to be a known AGN, 2MASX J123204981056010. These data make up the GRB150101B light curve on the XRT public archive (Evans et al. 2007, 2009). Fong et al. (2016) present two X-ray points taken from the Chandra X-ray Observatory at $T=7.94$ d (ID: 16508492 ; PI: E. Troja) and $T=39.68$ d (ID: 16708496 , PI: A. Levan). However, it is not clear that these data points are in fact related to GRB150101B. Just as the Swift/XRT data points turned out to be due to an AGN, the two Chandra points may well be from AGN activity in the field. This all stems from the fact that GRB150101B was ground-detected and therefore lacked prompt XRT emission to provide a good localization. Fong et al. then assume that the jet break time $t_{j}>39.68 \mathrm{~d}$ implies a jet-break opening angle $\theta_{j} \gtrsim 9^{\circ}$, where

$$
\theta_{j}=9.5^{\circ} t_{j, d}^{3 / 8}(1+z)^{3 / 8} E_{\mathrm{K}, \mathrm{iso}, 52}{ }^{-1 / 8} n_{0}{ }^{1 / 8}
$$

(Sari et al. 1999, Frail et al. 2001), where $t_{j, d}$ is the jet break time in days, $E_{\mathrm{K}, \text { iso }, 52}$ is the isotropic equivalent energy in units of $10^{52} \mathrm{erg}$, and $n_{0}$ is the circumstellar number density. Given (i) the problematic nature of the association of the X-ray flux with GRB150101B and (ii) the questionable 


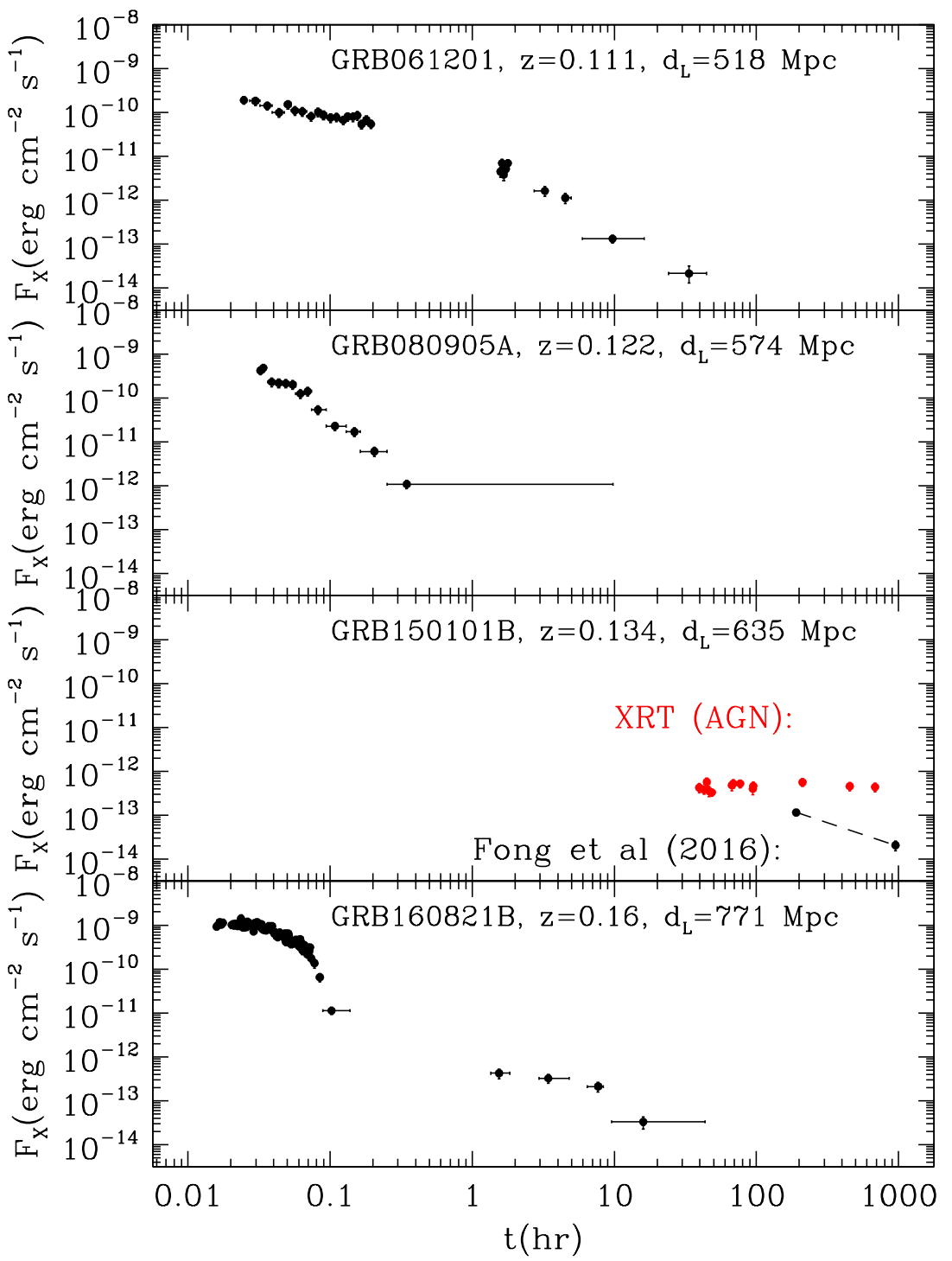

Figure 10: Early-time X-ray emission for a sampling of sGRBs with secure redshifts. For comparison GRB150101B which was a ground-detected Swift/BAT sGRB and therefore has an uncertain $z$ is shown on the same scale. The data in panels 1, 2, and 4, and the red points in panel 3 are $0.3-10 \mathrm{keV} \mathrm{Swift/XRT} \mathrm{data}$ (Evans et al. 2007, 2009) taken from the public web site. The two black points in the third panel indicate Chandra observations by E. Troja and A. Levan (presented in Fong et al. 2016) which could possibly be associated with GRB150101B. 
assumption of deriving a jet opening angle from two data points, this constraint on opening angle seems highly questionable.

\section{Conclusion}

GRBs are the brightest and most powerful EM transients. A modern suite of rapid response space and ground telescopes are providing important observations of the early emission. GRBs are caused by two very different types of sources: merging neutron stars (sGRBs) and collapse of massive stars (GRBs). Gravitational wave events from NS-NS mergers in coincidence with GRBs will prove the merger model.

\section{References}

[1] Abbott, B. P., Abbott, R., Abbott, T. D., et al. 2016a, Ph. Rev. Lett., 116, 061102

[2] Abbott, B. P., Abbott, R., Abbott, T. D., et al. 2016b, Ph. Rev. Lett., 116, 241102

[3] Atwood, W. B., Abdo, A. A., Ackermann, M., et al. 2009, ApJ, 697, 1071

[4] Barnes, J., \& Kasen, D. 2013, ApJ, 775, 18

[5] Barthelmy, S. D., Barbier, L. M., Cummings, J. R., et al. 2005, Space Sci. Rev., 120, 143

[6] Berger, E., Fong, W., \& Chornock, R. 2013, ApJL, 774, L23

[7] Berger, E., Penprase, B. E., Cenko, S. B., et al. 2006, ApJ, 642, 979

[8] Bloom, J. S., Perley, D. A., Li, W., et al. 2009, ApJ, 691, 723

[9] Bouwens, R. J., Illingworth, G. D., Franx, M., \& Ford, H. 2008, ApJ, 686, 230

[10] Bromm, V., \& Loeb, A. 2002, ApJ, 575, 111

[11] Burrows, D. N., Grupe, D., Capalbi, M., et al. 2006, ApJ, 653, 468

[12] Burrows, D. N., Hill, J. E., Nousek, J. A., et al. 2005, Space Sci. Rev., 120, 165

[13] Chen, H.-W., Prochaska, J. X., Bloom, J. S., \& Thompson, I. B. 2005, ApJL, 634, L25

[14] Chevalier, R. A., \& Li, Z.-Y. 2000, ApJ, 536, 195

[15] Ciardi, B., \& Loeb, A. 2000, ApJ, 540, 687

[16] Cucchiara, A., Levan, A. J., Fox, D. B., et al. 2011, ApJ, 736, 7

[17] East, W. E., \& Pretorius, F. 2012, ApJL, 760, L4

[18] Eichler, D., Livio, M., Piran, T., \& Schramm, D. N. 1989, Nature, 340, 126

[19] Evans, P. A., Beardmore, A. P., Page, K. L., et al. 2007, A\&A, 469, 379

[20] Evans, P. A., Beardmore, A. P., Page, K. L., et al. 2009, MNRAS, 397, 1177

[21] Fernández, R., Kasen, D., Metzger, B. D., \& Quataert, E. 2015, MNRAS, 446, 750

[22] Fernández, R., \& Metzger, B. D. 2013, MNRAS, 435, 502 
[23] Fong, W., Berger, E., Margutti, R., et al. 2012, ApJ, 756, 189

[24] Fong, W., Berger, E., Metzger, B. D., et al. 2014, ApJ, 780, 118

[25] Fong, W., Margutti, R., Chornock, R., et al. 2016, ApJ, 833, 151

[26] Fontes, C. J., Fryer, C. L., Hungerford, A. L., et al. 2017, astro-ph/1702.02990

[27] Foucart, F., O’Connor, E., Roberts, L., et al. 2015, PhRvD, 91, 124021

[28] Frail, D. A., Kulkarni, S. R., Sari, R., et al. 2001, ApJL, 562, L55

[29] Freiburghaus, C., Rosswog, S., \& Thielemann, F.-K., 1999, ApJL, 525, L121

[30] Fynbo, J. P. U., Starling, R. L. C., Ledoux, C., et al. 2006, A\&A, 451, L47

[31] Gehrels, N., Barthelmy, S. D., Burrows, D. N., et al. 2008, ApJ, 689, 1161

[32] Gehrels, N., Chincarini, G., Giommi, P., et al. 2004, ApJ, 611, 1005

[33] Gehrels, N., Ramirez-Ruiz, E., \& Fox, D. B. 2009, ARA\&A, 47, 567

[34] Goriely, S., Bauswein, A., \& Janka, H.-T., 2011, ApJL, 738, L32

[35] Grossman, D., Korobkin, O., Rosswog, S., \& Piran, T. 2014, MNRAS, 439, 757

[36] Grupe, D., Burrows, D. N., Patel, S. K., et al. 2006, ApJ, 653, 462

[37] Harrison, R., \& Kobayashi, S. 2013, ApJ, 772, 101

[38] Hopkins, A. M., \& Beacom, J. F. 2006, ApJ, 651, 142

[39] Hotokezaka, K., Kiuchi, K., Kyutoku, K., et al. 2013, PhRvD, 87, 024001

[40] Kasen, D., Badnell, N. R., \& Barnes, J. 2013, ApJ, 774, 25

[41] Kasen, D., Fernández, R., \& Metzger, B. D., 2015, MNRAS, 450, 1777

[42] Kawai, N., Kosugi, G., Aoki, K., et al. 2006, Nature, 440, 184

[43] Kistler, M. D., Yüksel, H., Beacom, J. F., et al. 2009, ApJL, 705, L104

[44] Kobayashi, S., \& Zhang, B. 2003a, ApJL, 582, L75

[45] Kobayashi, S., \& Zhang, B. 2003b, ApJ, 597, 455

[46] Kouveliotou, C., Meegan, C. A., Fishman, G. J., et al. 1993, ApJL, 413, L101

[47] Kulkarni, S. R. 2005, astro-ph/0510256

[48] Kyutoku, K., Ioka, K., \& Shibata, M. 2013, PhRvD, 88, 041503

[49] Lamb, D. Q. 2002, in Lighthouses of the Universe: The Most Luminous Celestial Objects and Their Use for Cosmology, Proc. MPA/ESO, 157

[50] Lamb, D. Q., \& Reichart, D. E. 2000, ApJ, 536, 1

[51] Laskar, T., Berger, E., Zauderer, B. A., et al. 2013, ApJ, 776, 119

[52] Li, L.-X., \& Paczyński, B. 1998, ApJ, 507, L59

[53] Madau, P., Haardt, F., \& Rees, M. J. 1999, ApJ, 514, 648

[54] Meegan, C., Lichti, G., Bhat, P. N., et al. 2009, ApJ, 702, 791

[55] Metzger, B. D., \& Berger, E. 2012, ApJ, 746, 48 
[56] Metzger, B. D., Martínez-Pinedo, G., Darbha, S., et al. 2010, MNRAS, 406, 2650

[57] Metzger, B. D., Piro, A. L., \& Quataert, E. 2008, MNRAS, 390, 781

[58] Ota, K., Iye, M., Kashikawa, N., et al. 2008, ApJ, 677, 12

[59] Piran, T., \& Fan, Y.-Z. 2007, Phil. Trans. Roy. Soc. A, 365, 1151

[60] Piran, T., Nakar, E., \& Rosswog, S. 2013, MNRAS, 430, 2121

[61] Prochaska, J. X., Gawiser, E., Wolfe, A. M., Castro, S., \& Djorgovski, S. G. 2003, ApJL, 595, L9

[62] Racusin, J. L., Karpov, S. V., Sokolowski, M., et al. 2008, Nature, 455, 183

[63] Robertson, B. E., \& Ellis, R. S. 2012, ApJ, 744, 95

[64] Roming, P. W. A., Kennedy, T. E., Mason, K. O., et al. 2005, Space Sci. Rev., 120, 95

[65] Salvaterra, R., Della Valle, M., Campana, S., et al. 2009, Nature, 461, 1258

[66] Sari, R., \& Piran, T. 1999a, ApJL, 517, L109

[67] Sari, R., \& Piran, T. 1999b, ApJ, 520, 641

[68] Sari, R., Piran, T., \& Halpern, J. P., 1999, ApJL, 519, L17

[69] Sari, R., Piran, T., \& Narayan, R. 1998, ApJL, 497, L17

[70] Savaglio, S. 2006, New Jour. Phys., 8, 195

[71] Savaglio, S., Glazebrook, K., \& LeBorgne, D. 2009, ApJ, 691, 182

[72] Sekiguchi, Y., Kiuchi, K., Kyutoku, K., \& Shibata, M. 2015, PhRvD, 91, 064059

[73] Sneden, C., Cowan, J. J., \& Gallino, R. 2008, ARAA, 46, 241

[74] Soderberg, A. M., Chandra, P., Frail, D., 2008, GCN, 7506, 1

[75] Surman, R., Mumpower, M. Cass, J., et al. 2014, in INPC 2013 - International Nuclear Physics Conference, ed. S. Lunardi et al, 66, 07024

[76] Tanaka, M., \& Hotokezaka, K. 2013, ApJ, 775, 113

[77] Tanaka, M., Hotokezaka, K., Kyutoku, K., et al. 2014, ApJ, 780, 31

[78] Tanvir, N. R., Fox, D. B., Levan, A. J., et al. 2009, Nature, 461, 1254

[79] Tanvir, N. R., Levan, A. J., Fruchter, A. S., et al. 2013, Nature, 500, 547

[80] Thöne, C. C., Fynbo, J., Goldoni, P., et al. 2011, Astron. Nach., 332, 281

[81] Trenti, M., Perna, R., \& Jimenez, R. 2015, ApJ, 802, 103

[82] Vestrand, W. T., Wren, J. A., \& Panaitescu, A., et al. 2014, Science, 343, 38

[83] Wanajo, S., Sekiguchi, Y., Nishimura, N., et al. 2014, ApJL, 789, L39

[84] Xu, D., de Ugarte Postigo, A., Leloudas, G., et al. 2013, ApJ, 776, 98

[85] Zhang, B., \& Kobayashi, S., 2005, ApJ, 2005, 628, 315

[86] Zou, Y. C., Wu, X. F., \& Dai, Z. G. 2005, MNRAS, 363, 93 